\title{
Effect of Static Stretching with Superficial Cooling on Muscle Stiffness
}

\section{(ㄷ)(1) (우 $\Theta$}

\author{
Authors \\ Masatoshi Nakamura', Ryo Hirabayashi' ${ }^{1}$, Shuhei Ohya², Takafumi Aoki², Daichi Suzuki², Mitsuki Shimamoto², \\ Takanori Kikumoto ${ }^{3}$, Wataru Ito ${ }^{3}$, Emi Nakamura', Tomoya Takabayashi'1, Mtsuaki Edama²
}

\author{
Affiliations \\ 1 Institute for Human Movement and Medical Sciences, \\ Niigata University of Health and Welfare, Niigata, Japan \\ 2 Department of Physical Therapy, Niigata University of \\ Health and Welfare, Niigata, Japan \\ 3 Physical Therapy, Niigata University of Health and \\ Welfare, Niigata, Japan
}

Key words

superficial cooling, shear elastic modulus, range of motion, stretch tolerance

$\begin{array}{ll}\text { received } & 20.03 .2018 \\ \text { revised } & 26.05 .2018 \\ \text { accepted } & 01.07 .2018\end{array}$

Bibliography
DOI https://doi.org/10.1055/a-0684-9375

Sports Medicine International Open 2018; 2: E142-E147

(c) Georg Thieme Verlag KG Stuttgart · New York

ISSN 2367-1890

\section{Correspondence}

Mr. Masatoshi Nakamura

Niigata University of Health and Welfare

Institute for Human Movement and Medical Sciences

1398 Shimami-cho, Kita-ku, Niigata City

\author{
950-3198 Niigata \\ Japan \\ Tel.: + 81/25/257 4451, Fax: + 81/25/2574451 \\ masatoshi-nakamura@nuhw.ac.jp
}

\section{ABSTRACT}

This study aimed to clarify the acute effect of static stretching (SS) with superficial cooling on dorsiflexion range of motion (DF ROM) and muscle stiffness. Sixteen healthy males participated in the cooling condition and a control condition in a random order. The DF ROM and the shear elastic modulus of medial gastrocnemius (MG) in the dominant leg were measured during passive dorsiflexion. All measurements were performed prior to (PRE) and immediately after 20 min of cooling or rested for $20 \mathrm{~min}$ (POST), followed by 2 min SS (POST SS). In cooling condition, DF ROM at POST and POST SS were significantly higher than that at PRE and DF ROM at POST SS was significantly higher than that at POST. In addition, the shear elastic modulus at POST was significantly higher than that at PRE and the shear elastic modulus at POST SS was significantly lower than those at PRE and POST. However, there were no significant differences in the percentage changes between PRE and POST SS between the cooling and control conditions. Our results showed that effects of SS with superficial cooling on increases in ROM and decrease in muscle stiffness were no more beneficial than those of SS alone.

\section{Introduction}

The application of thermal agents such as heat, ultrasound, and ice is popular treatment in rehabilitation and sports settings. Changes in tissue temperature due to thermal agents are believed to cause changes in metabolism, nerve transmission, hemodynamics, and mechanical properties $[5,6]$. In particular, tissue cooling by the application of ice (i. e., cryotherapy) is expected to have an analgesic effect, inhibiting inflammation and tissue metabolism. Therefore, cooling by ice or other forms is the preferred method of injury management for muscle strain, damage, and bruising [5]. In addition, cooling has been reported to be useful for recovery after exercise [7,29]. Thus, cooling is assumed to be one of the most effective treatments in rehabilitation and sports settings.

It is commonly believed that decreased flexibility (i. e., range of motion: ROM) is a harmful effect of cooling. In fact, previous studies investigating the effect of cooling on passive properties and muscle stiffness have reported that passive torque and muscle stiffness increased after cooling $[4,21,25]$. However, previous studies reported that ROM increased after cooling [18, 23]. Therefore, there is a discrepancy between the effect of cooling on ROM and muscle stiffness or passive torque. An increase in ROM may be caused not only by decreased passive torque and muscle stiffness but also changes in stretch tolerance because of changes in senso- 
ry mechanisms for pain and discomfort [20,31]. Furthermore, the previous study reported the change in stretch tolerance after cooling because of the analgesic effect of cooling [24]. Therefore, it is assumed that the increase in ROM after cooling could be involved in the change in stretch tolerance and not in the decrease in muscle stiffness.

In addition, previous studies have investigated the effect of stretching with superficial cooling, hypothesizing that the analgesic effect of cooling would increase the effect of stretching more than stretching alone. The results showed that there were no significant differences in terms of ROM gains between stretching with superficial cooling and stretching alone $[13,28]$, whereas greater ROM gains in stretching with superficial cooling were shown than those in stretching alone $[8,15]$. Thus, the effectiveness of stretching with superficial cooling relative to stretching alone has not yet been established. However, to the best of our knowledge, there have been no studies showing harmful effects of stretching with superficial cooling on ROM.

Meanwhile, as described above, passive torque and muscle stiffness increased after cooling [4, 21, 25]. Increased muscle stiffness and passive torque showed higher mechanical stress during passive stretching and reduced capacity of the muscle to withstand active or passive stretching [25]. Thus, increased muscle stiffness may increase the risk of muscle strain and damage [30]. Considering both of these points, it seems reasonable to assume that stretching with superficial cooling may not be effective in reducing muscle stiffness, despite there being no studies that have investigated this. Moreover, previous studies have reported that an increase in ROM may be caused not only by decreased passive torque and muscle stiffness but also changes in stretch tolerance [20, 31]. Therefore, it is necessary to investigate the effect of static stretching (SS) with superficial cooling not only on ROM but also on muscle stiffness and stretch tolerance. However, whether changes in muscle stiffness and stretch tolerance after SS with superficial cooling lead to ROM gains remains unclear. Therefore, this study aimed to clarify the acute effect of SS with superficial cooling on ROM, stretch tolerance, and muscle stiffness. The hypothesis of this study was that changes in stretch tolerance caused by superficial cooling would lead to increased stretching intensity, resulting in large ROM gains and decreased muscle stiffness.

\section{Material and Methods}

\section{Subjects}

Sixteen healthy males volunteered to participate in the study (mean \pm SD: aged $20.9 \pm 0.3$ years; height, $171.5 \pm 5.6 \mathrm{~cm}$; weight, $66.2 \pm 5.3 \mathrm{~kg}$ ). We excluded subjects with a history of neuromuscular disease and/or musculoskeletal injury involving the lower extremities. All subjects were fully informed of the procedures and purpose of the study, and all provided written informed consent. The study was approved by the Ethics Committee of Niigata University of Health and Welfare, Niigata, Japan (17677). This study was also performed in accordance with the ethical standards of the International Journal of Sports Medicine [11].

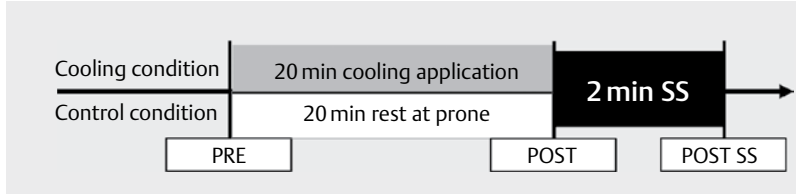

- Fig. 1 Experimental protocol of this study. SS: static stretching

\section{Experimental protocol}

A randomized repeated-measures experimental design was used to investigate the acute effects of SS with superficial cooling on DF ROM, passive torque at DF ROM, and muscle stiffness of medial gastrocnemius (MG) in the dominant leg (the preferred leg for kicking a ball). All subjects participated in the cooling condition and a control condition in a random order for at least 2 days, with no longer than a 1-week interval between both conditions. As shown in - Fig. 1, in the cooling condition, all measurements were performed prior to (PRE) and immediately after 20 min of cooling (POST), followed by 2 min SS (POST SS). In the control condition, subjects rested in the same position as the cooling condition for $20 \mathrm{~min}$ (POST) after the PRE measurement; SS intervention was then performed for 2 min (POST SS). The subjects were familiarized with the procedure and instructed to remain relaxed throughout the measurement period.

\section{Assessment of dorsiflexion range of motion and passive torque}

The subjects were instructed to lie in the prone position on a Biodex System 4.0 (Biodex Medical Systems Inc., Shirley, NY, USA) with their hips securely held in place with an adjustable lap belt. The knee of the dominant leg was kept in full extension, and the foot of the same leg was securely attached to the dynamometer footplate with adjustable lap belts. The footplate of the dynamometer was manually moved by an examiner slowly to avoid stretch reflex starting from an ankle angle of $30^{\circ}$ plantarflexion to the dorsiflexion angle just before the subjects started to feel discomfort or pain. The subjects were instructed to verbally inform the examiner when they started to feel discomfort or pain, and the angle just before this point was defined as dorsiflexion range of motion (DF ROM) $[2,3]$. In addition, passive torque at DF ROM was defined using the index of sensory perception, and an increase in passive torque at DF ROM was defined as a change in stretch tolerance (sensory perception) for passive stretching $[19,20]$.

\section{Assessment of shear elastic modulus}

In this study, we adopted the shear elastic modulus measured by ultrasonic shear wave elastography (SWE) as an index of muscle stiffness. The shear elastic modulus of MG was measured using ultrasonic SWE (Aplio 500, Toshiba Medical Systems, Tochigi, Japan) with a 5-14 MHz linear probe at $10^{\circ}$ dorsiflexion, similar to the conditions during the DF ROM and passive torque measurements. Based on the method used in previous studies [1,3], the shear elastic modulus of MG was measured at the proximal $30 \%$ of the lower leg length (from the popliteal crease to the lateral malleolus). An ultrasound transducer was positioned on the measurement points parallel to the direction of the muscle fibers, which were confirmed by tracing several fascicles without interruption across the B-mode image. 
In the present study, the quadrangular region of interest (ROI) was set to cover the whole muscle. The obtained elastographic images were analyzed using image analysis software (MSI Analyzer version 5.0; Institute of Rehabilitation Science, Tokuyukai Medical Corporation, Japan). The quadrangular ROI was set to be as large as possible within the color-coded area of the elastographic images while accounting for the artifact from aponeurosis ( $>$ Fig. 2). The average value of Young's modulus in the quadrangular ROI was automatically calculated in this software. On the basis of previous studies [12,27], the shear elastic modulus was calculated by dividing the obtained Young's modulus by three. The elastographic image of MG was measured twice, and the average value of the shear elastic modulus was used for further analysis [2, 3, 22].

\section{Cooling condition}

The subjects received a bag containing ice cubes in the prone position with the knee extended, similar to the conditions during the DR ROM and shear elastic modulus measurements in the cooling condition. The ice bag was secured to the whole MG for $20 \mathrm{~min}$, in accordance with previous studies $[9,15]$.

\section{Static stretching}

The SS intervention was performed using a dynamometer in the prone position with the knee extended, similar to the conditions during the DR ROM and shear elastic modulus measurements. The footplate of the dynamometer was manually moved by an examiner, starting from $30^{\circ}$ plantarflexion to DF ROM, and the ankle joint was held at DF ROM for 2 min, which has been reported to decrease the shear elastic modulus [22].

\section{Assessment of skin temperature}

Skin temperature of MG in the cooling condition was measured at PRE, POST, and POST SS using an infrared thermometer (73010, Shinwa Rules Co., Ltd, Niigata, Japan). Skin temperature was $32.4^{\circ} \mathrm{C} \pm 1.5^{\circ} \mathrm{C}$

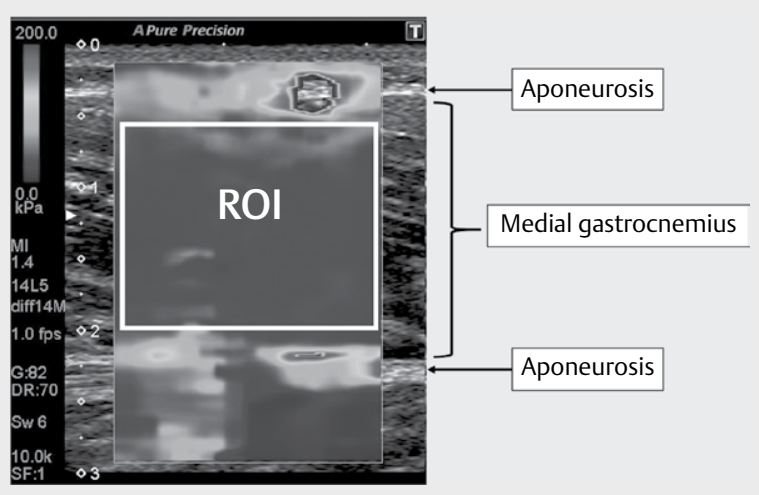

-Fig. 2 Typical example of measurement of shear elastic modulus by ultrasonic shear wave elastographic imaging. The quadrangular region of interest (ROI) within the color-coded area of the elastographic images was set, and the average value of Young's modulus in the quadrangular ROI was automatically calculated. In addition, the shear elastic modulus was calculated by dividing the obtained Young's modulus by three. at PRE, whereas skin temperature was $16.0^{\circ} \mathrm{C} \pm 4.2^{\circ} \mathrm{C}$ at POST and $23.9^{\circ} \mathrm{C} \pm 2.5^{\circ} \mathrm{C}$ at POST SS. Statistical analysis showed that the POST and POST SS skin temperatures were significantly lower than that of PRE, which confirmed that MG was cooled during the measurements of DF ROM, passive torque, and shear elastic modulus of MG.

\section{Inter-day reliability of measurements of shear elastic modulus}

To assess the inter-day reliability of the measurements of shear elastic modulus, these measurements were done twice on two different days by the same investigator with a 2-day to 1-week interval between the two sessions for ten participants (mean \pm SD: age, $20.9 \pm 0.3$ years; height, $170.3 \pm 4.7 \mathrm{~cm}$; weight, $63.4 \pm 2.6 \mathrm{~kg}$ ).

\section{A priori sample size calculation}

We calculated the sample size needed for a repeated two-way analysis of variance $(A N O V A$ ) (alpha error $=0.05$; power $=0.80$; effect size $=0.4$ [large]); the requisite number of participants for this study was 12 in each condition. We chose a large effect size on the basis of a previous study [2].

\section{Statistical analysis}

SPSS (version 24.0; SPSS Japan Inc., Tokyo, Japan) was used for statistical analyses. The inter-day reliability of the measurement of shear elastic modulus was assessed using the intraclass correlation coefficient (ICC $[1,1])$. For all variables, a repeated two-way ANOVA using two factors [condition (cooling vs. control condition) and test time (PRE vs. POST vs. POST SS)] was used to analyze the interaction and main effect. When a significant interaction was observed, the Bonferroni multiple comparison test was used to determine the differences among the PRE, POST, and POST SS variables in both the cooling and control conditions. In addition, the paired t-test was used to compare the percentage change from PRE to POST SS between the cooling and control conditions to investigate the combinational effect of SS with superficial cooling. The percentage change was calculated using the shear elastic modulus in PRE and POST SS in each condition, using the following equation:

Percentage change $=($ PRE value - POST SS value $) /($ PRE value $) \times 100$ The statistical significance was set at an alpha level of 0.05 .

\section{Results}

\section{Inter-day reliability of measurement of shear elastic modulus}

The ICC $(1,1)$ for the shear elastic modulus measurement was 0.919 (trial 1: 18.9 $\pm 11.4 \mathrm{kPa}$; trial 2: $19.4 \pm 9.1 \mathrm{kPa}$ ).

\section{Changes in DF ROM and passive torque at DF ROM}

DF ROM and passive torque at DF ROM in both the cooling and control conditions are presented in $>$ Table 1. The two-way ANOVA indicated a significant interaction $(p<0.01)$ and main effect for test time $(p<0.01)$ for DF ROM. In the cooling condition, a post hoc test revealed that DF ROM at POST and POST SS were significantly higher than that at PRE and that DF ROM at POST SS was significantly 
- Table 1 Changes in DF ROM, passive torque at DF ROM, and shear elastic modulus of MG.

\begin{tabular}{|c|c|c|c|c|c|c|c|c|}
\hline & \multicolumn{3}{|c|}{ Cooling condition } & \multicolumn{3}{|c|}{ Control condition } & \multicolumn{2}{|c|}{ Interaction effect } \\
\hline & PRE & POST & POST SS & PRE & POST & POST SS & $p$ value & partial $\eta^{2}$ \\
\hline DF ROM (degree) & $36.2 \pm 4.8$ & $40.4 \pm 5.1^{* *}$ & $43.0 \pm 6.2^{* *} \# \#$ & $37.0 \pm 4.2$ & $36.6 \pm 3.9$ & $41.8 \pm 4.7^{* *} \# \#$ & $P<0.01$ & 0.506 \\
\hline $\begin{array}{l}\text { Passive torque at DF } \\
\operatorname{ROM}(\mathrm{Nm})\end{array}$ & $24.5 \pm 4.7$ & $30.1 \pm 7.4^{* *}$ & $32.1 \pm 8.9 * *$ & $24.8 \pm 4.3$ & $23.4 \pm 4.6$ & $29.4 \pm 7.7^{* *} \# \#$ & $P<0.01$ & 0.409 \\
\hline $\begin{array}{l}\text { Shear elastic } \\
\text { modulus of } \mathrm{MG}(\mathrm{kPa})\end{array}$ & $15.9 \pm 7.2$ & $21.3 \pm 8.3^{*}$ & $10.1 \pm 5.5^{* *} \# \#$ & $17.0 \pm 9.5$ & $18.0 \pm 9.8$ & $11.1 \pm 5.6^{* *} \# \#$ & $P=0.014$ & 0.247 \\
\hline
\end{tabular}

- Table 2 Comparison of the percent changes between cooling condition and control condition.

\begin{tabular}{|l|l|l|l|}
\hline $\begin{array}{l}\text { The percent change between } \\
\text { PRE and POST SS (\%) }\end{array}$ & $\begin{array}{l}\text { Cooling } \\
\text { condition }\end{array}$ & $\begin{array}{l}\text { Control } \\
\text { condition }\end{array}$ & P value \\
\hline DF ROM & $18.9 \pm 6.9$ & $13.3 \pm 10.0$ & $\mathrm{P}=0.077$ \\
\hline passive torque at DF ROM & $30.3 \pm 21.8$ & $18.4 \pm 18.4$ & $\mathrm{P}=0.152$ \\
\hline shear elastic modulus of MG & $-35.5 \pm 17.5$ & $-29.1 \pm 26.4$ & $\mathrm{P}=0.391$ \\
\hline SS; static stretching, ROM; range of motion, MG; medial gastrocnemius \\
\hline
\end{tabular}

higher than that at POST. In addition, in the control condition, the post hoc test revealed that DF ROM at POST SS was significantly higher than those at PRE and POST.

Moreover, the two-way ANOVA indicated a significant interaction $(p<0.01)$ and main effects for condition and test time (condition: $p=0.012$; test time: $p<0.01$, respectively) for passive torque at $D F$ ROM. In the cooling condition, the post hoc test revealed that DF ROM at POST and POST SS was significantly higher than that at PRE, whereas there was no significant difference between POST and POST SS. In addition, in the control condition, the post hoc test revealed that DF ROM at POST SS was significantly higher than those at PRE and POST.

\section{Changes in shear elastic modulus of MG}

The shear elastic modulus of MG in both the cooling and control conditions is presented in $>$ Table 1. The two-way ANOVA indicated a significant interaction $(p=0.014)$ and main effect for test time $(p<0.01)$.

In the cooling condition, the post hoc test revealed that the shear elastic modulus of MG at POST was significantly higher than that at PRE and the shear elastic modulus at POST SS was significantly lower than those at PRE and POST. In addition, in the control condition, the post hoc test revealed that the shear elastic modulus at POST SS was significantly lower than those at PRE and POST.

\section{Comparison of the percentage changes between the cooling and control conditions}

The percentage changes between PRE and POST SS are presented in $>$ Table 2. For all variables, there were no significant differences between the cooling and control conditions.

\section{Discussion}

In the present study, we investigated the effect of SS with superficial cooling, not only on ROM but also on muscle stiffness and stretch tolerance, and investigated the effectiveness of stretching with superficial cooling. There were three major findings. First, DF ROM, passive torque at DF ROM, and muscle stiffness increased after 20 min of superficial cooling. Second, DF ROM and passive torque at DF ROM significantly increased and muscle stiffness decreased after SS with superficial cooling. Third, there were no significant differences in the percentage changes between PRE and POST SS for all variables between the cooling and control conditions. To the best of our knowledge, this is the first report to investigate the effect of SS with superficial cooling, not only on ROM but also on muscle stiffness and stretch tolerance.

In the present study, DF ROM significantly increased after superficial cooling, which is consistent with the results of previous studies $[18,24]$. The increase in ROM has been assumed to be involved in changes in stretch tolerance because of the analgesic effect of cooling $[8,24]$. In the present study, passive torque at DF ROM, as an index of stretch tolerance, significantly increased after superficial cooling, which is consistent with the results of previous studies $[8,24]$. In addition, muscle stiffness significantly increased after superficial cooling, which is also consistent with the results of a previous study [25]. Increased muscle stiffness and passive torque showed higher mechanical stress applied by passive stretching, and the capacity of the muscle to withstand active or passive stretching reduced [25]. Thus, increased muscle stiffness may increase the risk of muscle strain and damage [30]. Moreover, many previous studies have observed that muscle strength and performance declined after cooling $[14,26]$. Considering our results and those of previous studies, it is assumed that increased muscle stiffness and decreased performance after cooling may lead to a high risk of injury. Therefore, athletes should not return to play immediately after cooling. However, the cooling duration used in the present study was $20 \mathrm{~min}$, which may be longer than that commonly used in clinical and sports settings. Thus, future research is needed to investigate the effect of superficial cooling on muscle stiffness and performance with the cooling duration used in clinical and sports settings.

In the cooling condition, DF ROM increased and muscle stiffness significantly decreased immediately after SS with superficial cooling, whereas there was no significant change in passive torque at DF ROM. Meanwhile, DF ROM and passive torque at DF ROM increased and muscle stiffness significantly decreased immediately after $\mathrm{SS}$ in the control condition. These results indicate that the increase in DF ROM was contributed to by both the decrease in muscle stiffness and the change in stretch tolerance after SS intervention only. However, the increase in DF ROM in the cooling condition was contributed to not by the change in stretch tolerance but by 
the decrease in muscle stiffness. In addition, there were no significant differences in the percentage changes between PRE and POST SS for all variables between the cooling and control conditions. Therefore, the results did not support our hypothesis that the magnitude of changes in DF ROM and muscle stiffness in the cooling condition would be larger than those in the control condition. However, the results were consistent with those of previous studies in that there were no significant differences in ROM gains between stretching with superficial cooling and stretching alone [13, 28]. Thus, as described above, muscle stiffness increased immediately after cooling, whereas there was no significant percentage change in muscle stiffness between the cooling and control conditions. It was assumed that these results are related to the analgesic effect of cooling. A previous study reported that the higher the applied stretching intensity, the greater the decrease in muscle stiffness [10]. In the present study, because stretch tolerance, shown by the significant increase in the passive torque at DF ROM, changed after cooling (cooling condition: $30.1 \pm 7.4 \mathrm{Nm}$ vs. control condition: $23.4 \pm 4.6 \mathrm{Nm}, \mathrm{p}<0.01$ ), SS intervention was higher in the cooling condition than in the control condition. Although muscle stiffness temporally increased after cooling, there was a large decrease in muscle stiffness following SS intervention by higher stretching intensity. Therefore, it was assumed that the magnitude of the decrease in muscle stiffness in the cooling condition was similar to that in the control condition.

A previous study reported that subjects with tight hamstrings show lower stretch tolerance compared to those with normal hamstrings [16]. In addition, a study has reported that stretch tolerance decreases in muscles that suffer from eccentric exercise-induced damage [17]. Our results showed that SS intervention with higher stretching intensity could be performed because cooling changed the stretch tolerance, whereas there was an adverse effect of increased muscle stiffness. Therefore, it could be expected that ROM and muscle stiffness in subjects with tight hamstrings, and subjects who suffer from delayed muscle pain, could be more effectively improved by stretching with superficial cooling than by SS intervention alone. Therefore, it is necessary to investigate the effect of SS with superficial cooling in subjects with decreased stretch tolerance due to tight hamstrings or muscle damage.

In the present study, we measured skin and not muscle temperature, which is a limitation. Future studies are needed to investigate the relationship between changes in muscle temperature and muscle stiffness after SS with superficial cooling.

\section{Conclusion}

In this study, we investigated the acute effect of SS with superficial cooling on ROM, stretch tolerance, and muscle stiffness in the present study. Our results showed that muscle stiffness decreased immediately after SS with superficial cooling, whereas there was no significant difference in terms of the magnitude of the decrease in muscle stiffness between the cooling and control conditions. Therefore, we concluded that the effects of SS with superficial cooling on increases in ROM and decreases in muscle stiffness were no more beneficial than those of SS alone.

\section{Acknowledgements}

This work was supported by a Developed Research from the Niigata University of Health and Welfare (H29C18). In addition, the authors would like to thank Enago (http://www.enago.jp/) for editorial assistance with the manuscript.

\section{Conflict of Interest}

The authors declare that they have no conflict of interest.

\section{References}

[1] Akagi R, Chino K, Dohi M, Takahashi H. Relationships between muscle size and hardness of the medial gastrocnemius at different ankle joint angles in young men. Acta Radiol 2012; 53: 307-311

[2] Akagi R, Takahashi H. Acute effect of static stretching on hardness of the gastrocnemius muscle. Med Sci Sports Exerc 2013; 45: 1348-1354

[3] Akagi R, Takahashi H. Effect of a 5-week static stretching program on hardness of the gastrocnemius muscle. Scand J Med Sci Sports 2014; 24: 950-957

[4] Akehi K, Long BC, Warren AJ, Goad CL. Ankle joint angle and lower leg musculotendinous unit responses to cryotherapy. J Strength Cond Res 2016; 30: 2482-2492

[5] Bleakley C, McDonough S, MacAuley D. The use of ice in the treatment of acute soft-tissue injury: A systematic review of randomized controlled trials. Am J Sports Med 2004; 32: 251-261

[6] Bleakley CM, Costello JT. Do thermal agents affect range of movement and mechanical properties in soft tissues? A systematic review. Arch Phys Med Rehabil 2013; 94: 149-163

[7] Bleakley CM, Davison GW. What is the biochemical and physiological rationale for using cold-water immersion in sports recovery? A systematic review. Br J Sports Med 2010; 44: 179-187

[8] Brodowicz GR, Welsh R, Wallis ]. Comparison of stretching with ice, stretching with heat, or stretching alone on hamstring flexibility. J Athl Train 1996; 31: 324-327

[9] Dykstra JH, Hill HM, Miller MG, Cheatham CC, Michael T], Baker R]. Comparisons of cubed ice, crushed ice, and wetted ice on intramuscular and surface temperature changes. J Athl Train 2009; 44: 136-141

[10] Freitas SR, Andrade RJ, Larcoupaille L, Mil-homens P, Nordez A. Muscle and joint responses during and after static stretching performed at different intensities. Eur J Appl Physiol 2015; 115: 1263-1272

[11] Harriss D], Macsween A, Atkinson G. Standards for ethics in sport and exercise science research: 2018 update. Int J Sports Med 2017; 38: $1126-1131$

[12] Hirata K, Miyamoto-Mikami E, Kanehisa H, Miyamoto N. Muscle-specific acute changes in passive stiffness of human triceps surae after stretching. Eur J Appl Physiol 2016; 116: 911-918

[13] Knight CA, Rutledge CR, Cox ME, Acosta M, Hall SJ. Effect of superficial heat, deep heat, and active exercise warm-up on the extensibility of the plantar flexors. Phys Ther 2001; 81: 1206-1214

[14] Kubo K, Kanehisa H, Fukunaga T. Effects of cold and hot water immersion on the mechanical properties of human muscle and tendon in vivo. Clin Biomech 2005; 20: 291-300

[15] Larsen CC, Troiano JM, Ramirez RJ, Miller MG, Holcomb WR. Effects of crushed ice and wetted ice on hamstring flexibility. J Strength Cond Res 2015; 29: 483-488 
[16] Magnusson SP, Simonsen EB, Aagaard P, Boesen J, Johannsen F, Kjaer M. Determinants of musculoskeletal flexibility: Viscoelastic properties, cross-sectional area, EMG and stretch tolerance. Scand J Med Sci Sports 1997; 7: 195-202

[17] Matsuo S, Suzuki S, Iwata M, Hatano G, Nosaka K. Changes in force and stiffness after static stretching of eccentrically damaged hamstrings. Eur J Appl Physiol 2015; 115: 981-991

[18] Minton J. 1992 Student Writing Contest-1st runner-up: A comparison of thermotherapy and cryotherapy in enhancing supine, extended-leg, hip flexion. J Athl Train 1993; 28: 172-176

[19] Mitchell UH, Myrer JW, Hopkins JT, Hunter I, Feland JB, Hilton SC. Acute stretch perception alteration contributes to the success of the PNF "contract-relax" stretch. J Sport Rehabil 2007; 16: 85-92

[20] Mizuno T, Matsumoto M, Umemura Y. Viscoelasticity of the muscletendon unit is returned more rapidly than range of motion after stretching. Scand J Med Sci Sports 2013; 23: 23-30

[21] Muraoka T, Omuro K, Wakahara T, Muramatsu T, Kanehisa H, Fukunaga T, Kanosue K. Effects of muscle cooling on the stiffness of the human gastrocnemius muscle in vivo. Cells Tissues Organs 2008; 187: 152-160

[22] Nakamura M, Ikezoe T, Kobayashi T, Umegaki H, Takeno Y, Nishishita S, Ichihashi N. Acute effects of static stretching on muscle hardness of the medial gastrocnemius muscle belly in humans: An ultrasonic shear-wave elastography study. Ultrasound Med Biol 2014; 40: 1991-1997

[23] Newton RA. Effects of vapocoolants on passive hip flexion in healthy subjects. Phys Ther 1985; 65: 1034-1036
[24] Park KN, Kwon OY, Weon JH, Choung SD, Kim SH. Comparison of the effects of local cryotherapy and passive cross-body stretch on extensibility in subjects with posterior shoulder tightness. J Sports Sci Med 2014; 13: 84-90

[25] Point M, Guilhem G, Hug F, Nordez A, Frey A, Lacourpaille L. Cryotherapy induces an increase in muscle stiffness. Scand J Med Sci Sports 2018; 28: 260-266

[26] Pritchard KA, Saliba SA. Should athletes return to activity after cryotherapy? J Athl Train 2014; 49: 95-96

[27] Taniguchi K, Shinohara M, Nozaki S, Katayose M. Acute decrease in the stiffness of resting muscle belly due to static stretching. Scand J Med Sci Sports 2015; 25: 32-40

[28] Taylor BF, Waring CA, Brashear TA. The effects of therapeutic application of heat or cold followed by static stretch on hamstring muscle length. J Orthop Sports Phys Ther 1995; 21: 283-286

[29] Vaile J, Halson S, Gill N, Dawson B. Effect of hydrotherapy on the signs and symptoms of delayed onset muscle soreness. Eur J Appl Physiol 2008; 102: 447-455

[30] Watsford ML, Murphy AJ, McLachlan KA, Bryant AL, Cameron ML, Crossley KM, Makdissi M. A prospective study of the relationship between lower body stiffness and hamstring injury in professional Australian rules footballers. Am J Sports Med 2010; 38: 2058-2064

[31] Weppler CH, Magnusson SP. Increasing muscle extensibility: A matter of increasing length or modifying sensation? Phys Ther 2010; 90: 438-449 